\title{
The use of polymerase chain reaction in the diagnosis and management of prosthetic joint infections: a debatable issues at this time
}

\author{
Temi Ogunleye, Marlina Ponce de Leon, Suresh J. Antony*
}

\begin{abstract}
Department of of Medicine/Section of Infectious Diseases, Burrell College of Osteopathic Medicine, Las Cruces, New Mexico, U.S.A
\end{abstract}

Received: 17 November 2018

Revised: 18 January 2019

Accepted: 21 January 2019

\section{*Correspondence:}

Dr. Suresh J. Antony,

E-mail: suresh.antony@att.net

Copyright: ( $)$ the author(s), publisher and licensee Medip Academy. This is an open-access article distributed under the terms of the Creative Commons Attribution Non-Commercial License, which permits unrestricted non-commercial use, distribution, and reproduction in any medium, provided the original work is properly cited.

\begin{abstract}
Joint replacement surgery is increasing due to its success in decreasing pain and restoring function. Prosthetic joint infections (PJI) is one of the most detrimental complications of the surgery. These infections can either be acute or chronic and can be caused by a variety of organisms. Effective and efficient identification of the cause of infection is vital so that proper treatment can be provided. The use of polymerase chain reaction (PCR) is a possibility for diagnosis and management of PJI with a reduction in the use of incorrect antibiotics. This is due to its ability to quickly diagnosis viral, bacterial, rickettsia, mycobacterial, and protozoal infection in hours. It also has high sensitivity and specificity even with antimicrobial usage and biofilm production. However, more studies need to be done in order to be able to classify it as a possible gold standard.
\end{abstract}

Keywords: Joint replacement surgery, Prosthetic joint infections, Antibiotic usage, Polymerase chain reaction

\section{INTRODUCTION}

Joint replacement surgery results in improvement of quality of life by decreasing pain and restoring function with high success rates. High volume practices suggest that $85-90 \%$ of patients who undergo total joint replacements receive substantial pain relief and functional improvement. ${ }^{1,2}$ In 2010, 777,000 total knee arthroplasty and 280,000 total hip arthroplasty procedures were estimated in the United States. There is an estimated increase in demand for these procedures with an increase of around 65,000/year for a knee and 15,000/ year for hip. ${ }^{3}$ Failure rates of joint replacement are relatively low, about $1 \%$ per year, and $90 \%$ of postoperative patients still have a functioning joint 10 years later. ${ }^{3}$

The success rate for joint replacement surgeries is about $90 \%$, and a small percentage (1-2\%) of cases have complications ranging from ongoing pain, aseptic device failure loosening, and prosthetic joint infections (PJI). According to Crawford et al, hip replacement surgery has a failure rate of 1\%/year for the first 15 years that requires revision surgery. This failure is stated to be mostly due to aseptic loosening. ${ }^{4}$ PJIs are the most serious complication of joint arthroplasty. Acute infection following surgery usually occurs within 3 months with virulent organisms, such as staphylococcus aureus and gram negative bacilli that were inoculated at implantation. Less virulent organisms, such as coagulase negative Staphylococci and Propionibacterium acnes, tend to cause chronic infections that occur months/years after the surgery. ${ }^{5}$

It is important to state that culture negative prosthetic joint infections (CNPJIs) occur when there is no growth of microorganisms in cultures of infected peri-prosthetic tissue samples. Yoon Et al reported that the incidence of CNPJI was reported to range from $7.0 \%$ to $42.1 \%$. ${ }^{6}$ When 
a situation like this arises, it is important to determine whether the results are a true negative or false negative for example when there is fungi or mycobacterial infections or biofilm producing microorganisms that are not easily detected because they do not grow on routine media. ${ }^{6}$ Million et al reported that $46 \%$ of CNPIs were caused by fungi and $43 \%$ by mycobacteria. ${ }^{6}$ Treating these patients with CNPJI poses unique challenges due to lack of identification of pathogens. Treatment failure occurs due to lack of accurate diagnostic tools in PJI. Therefore, fungi, resistant pathogens, and fastidious pathogens are often missed and results in recurrent infections and treatment failures. Currently, the most appropriate surgical and medical treatments for CNPIs is not clear.
Procedures associated with prosthetic infections following joint replacement has a higher total number of stays in hospitals, total surgeries, and higher total hospitals costs. Complications related to PJI are polymicrobial due to multidrug resistant bacteria or difficult to treat microorganisms such as methicillin resistance staphylococci with some resistance to teicoplanin. ${ }^{5,7}$ There is importance to develop quick and effective methods of determining the cause of infections to avoid inappropriate use of antibiotics to reduce cost and further complications.

Table 1: Summarization of current methods of working up infectious joints.

\begin{tabular}{|c|c|c|}
\hline Tests & Advantage & Disadvantage \\
\hline $\begin{array}{l}\text { Blood tests } \\
\text { - ESR, CRP, WBC } \\
\text { count. } \\
\text { - Alpha defensins } \\
\end{array}$ & $\begin{array}{l}\text { Raises possibility an infection, } \\
\text { especially if persistently elevated } \\
\text { Higher sensitivity than ESR and CRP; } \\
\text { not affected by use of antimicrobials }\end{array}$ & $\begin{array}{l}\text { Not specific or sensitive, especially } \\
\text { post-op; affected by antimicrobial use }\end{array}$ \\
\hline Histopathology & $\begin{array}{l}\text { Can be used in conjunction with other } \\
\text { diagnostic measures to assess for PJI }\end{array}$ & $\begin{array}{l}\text { No guidelines on its use; requires tissue } \\
\text { sample }\end{array}$ \\
\hline Synovial fluid analysis & High sensitivity and specificity & $\begin{array}{l}\text { Wear and tear of aging leads to increased } \\
\text { bone resorption leading to false positives }\end{array}$ \\
\hline $\begin{array}{l}\text { Imaging } \\
\begin{array}{l}\text { - X-rays } \\
\text { - FDG PET } \\
\text { - Bone scintigraphy }\end{array}\end{array}$ & $\begin{array}{l}\text { Can aid in differentiating between aseptic } \\
\text { loosening and PJI } \\
\text { - Can Identify non-infectious causes } \\
\text { (fractures) } \\
\text { - } \quad \text { High specificity and sensitivity - } \\
\text { visualizes inflammatory cells that } \\
\text { utilize glucose } \\
\text { - Useful for PJI occurring later after } \\
\text { implantation }\end{array}$ & $\begin{array}{l}\text { - Not specific or sensitive } \\
\text { - High cost; not widely used } \\
\text { - Low specificity for PJIs that occur } \\
\text { acutely after implantation }\end{array}$ \\
\hline $\begin{array}{l}\text { Peri-prosthetic tissue } \\
\text { culturing }\end{array}$ & Gold standard & $\begin{array}{l}\text { Low specificity; doesn't detect fastidious } \\
\text { organisms or biofilm producing organisms } \\
\text { that grow on biofilms; takes days for } \\
\text { growth to return }\end{array}$ \\
\hline Sonication & $\begin{array}{l}\text { Increases sensitivity of cultures by } \\
\text { disrupting biofilms }\end{array}$ & $\begin{array}{l}\text { Low sensitivity with prior antimicrobial } \\
\text { therapy and infection by fastidious } \\
\text { organism }\end{array}$ \\
\hline DNA PCR & $\begin{array}{l}\text { Allows rapid diagnosis of viral, bacterial, } \\
\text { rickettsia, mycobacterial, and protozoal } \\
\text { infection in hours; high sensitivity and } \\
\text { specificity even with antimicrobial usage } \\
\text { and biofilm production }\end{array}$ & $\begin{array}{l}\text { False negatives occur under different } \\
\text { circumstances; results can be hard to } \\
\text { interpret; no guidelines on its use in PJI }\end{array}$ \\
\hline
\end{tabular}

\section{PURPOSE OF THIS DOCUMENT}

The goal of this document is to review PCR as a possible standard for diagnosing PJIs.

\section{RISK FACTORS}

It has been reported that $12-20 \%$ of all prostheses are found to be infected after primary revision surgeries. ${ }^{8-10}$ There are several demographic factors and co-morbid conditions that put patients at a higher risk for PJIs such an overweight male, age greater than 65 years, immunosuppression, and a history of previous revision. ${ }^{8,11,5}$ Additionally, other studies have shown that obesity and increasing BMI puts patients at a greater risk for infections. ${ }^{7} \mathrm{Wu}$ et al performed a study on patients that underwent total hip and knee arthroplasties and concluded that patients with a BMI greater than 28 $\mathrm{kg} / \mathrm{m}^{2}$ had a 2.77 -fold higher risk of PJI compared with patients with a BMI between 18.5 and $28 \mathrm{~kg} / \mathrm{m}^{2} .^{12}$ Another study performed by Peel and colleagues reported 
a $10 \%$ increase in risk of infection is associated with every $1 \mathrm{~kg} / \mathrm{m}^{2}$ increase in BMI. ${ }^{8,13,14}$

A second co-morbid condition that predisposes a patient to infection is diabetes. A study showed that diabetes mellitus and hyperglycemia prior to and after operation were predictors for postoperative infection following total joint arthroplasty. In the same study, Mraovic showed that patients without a diagnosis of diabetes who developed postoperative hyperglycemia had a significantly increased risk for the infection. ${ }^{15}$ However, in vitro studies have shown that hyperglycemia allows for increased formation of biofilms, but there is not a clear link to diabetes and PJIs. ${ }^{16,17}$

Prior antibiotic use, is one of the main risk factor for CNPJIs. According to Elie et al, use of antimicrobial therapy during the 3 months prior to the diagnosis of CNPJI was present in $53 \%$ patients and $23 \%$ of patients were receiving an antimicrobial agent up to the time of peri-prosthetic tissue culture. ${ }^{11}$ The usage of prior antimicrobial therapy and post-operative wound drainage after arthroplasty were associated with increased odds of PJI being culture negative", 5,18

\section{BIOFILMS}

Most infections associated with PJIs are related to pathogens that produce biofilms such as staphylococcus, fungi, and pseudomonas. These microorganisms organize themselves into a structured colony and surround themselves into a self-made extracellular matrix that allow them to adhere to surfaces such as implanted devices, prostheses, and damaged tissues. ${ }^{5}$ Organisms that are cultured included gram positive pathogens in $50 \%$ of the cases, of which staphylococcus aureus was identified in $33 \%$ of patients and coagulase negative staphylococcus in about $19 \%$ of isolates. ${ }^{8,9,10}$

\section{CLINICAL FEATURES OF PJI}

According to the Infectious Disease Society of America (IDSA), there are 3 situations in which one should suspect a PJI and these include (1) when there is a sinus tract or persistent wound drainage over a joint prosthesis, (2) acute onset of a pain in the prosthesis and (3) any chronic painful prosthesis. ${ }^{19}$ A study performed by Peal et al demonstrated that pain was reported in $42 \%$ of patients, while drainage from the surgical site was the most frequent finding in $72 \%$ of patients. ${ }^{13,17}$ These findings reveal that there is variation in the clinical presentation of PJIs. This is most likely due to differences in virulence of micro-organisms and varying host response to them.

PJIs also can be classified and grouped into three categories (1) early infections are those occurring within 3 months of implantation, (2) delayed when presenting between 3 and 24 months and 3.late if presenting beyond 2 years. $^{20}$ Early and delayed PJI are usually due to microorganisms that were introduced during surgery while late infections represent hematogenous spread of the microorganisms such as UTI, transient bacteremia, associated endocarditis, and other primary sources of infection. ${ }^{5}$ Initially PJI presents with acute inflammatory responses such as erythema, pain, and drainage at a site of implantation while late and chronic infections usually present with fewer signs of acute inflammation. The latter is often characterized by chronic pain and loosening of the implant, which may be visible, along with decreased range of motion. ${ }^{5}$ Loosening of prosthetic material alone is not enough to diagnose a late onset PJI because this can occur in absence of an infection.

\section{DIAGNOSTIC STUDIES}

The diagnosis of CNPIJIs can be difficult and is usually a combination of clinical signs and symptoms, blood tests, histopathology, radiological findings, and microbiological tests. ${ }^{9}$ Elie et al suggests that CNPJIs are diagnosed on the basis of one of the following: presence of purulence surrounding the prosthesis, histopathologic findings of acute inflammation of peri-prosthetic tissue samples, or a cutaneous sinus tract communicating with the prosthesis, in addition to there being no growth on aerobic and anaerobic cultures submitted to the clinical microbiology laboratory. ${ }^{11}$ The IDSA also includes synovial fluid with a leukocytosis and/or with the predominance of neutrophils or growth of identical micro-organism in at least two intraoperative cultures in their criteria for diagnostic criteria. ${ }^{19}$

Routine blood tests assess the host response to pathogens and may suggest an infection but these markers are nonspecific. For example, elevated C-reactive protein (CRP), leukocyte count, and erythrocyte sedimentation rate (ESR) may diagnose an infection but are unhelpful, especially post operatively because they will be elevated for 14 days after surgery. ${ }^{8,5}$ However, persistent and repetitive elevation of CRP and ESR together raises the possibility of an infected joint, even though they are not specific or sensitive. A blood test that is worth discussing is alpha defensins. Defensins, also known as human neutrophil peptide, are proteins that are normally released by neutrophils in response to pathogenic infections ${ }^{21}$. A recent study was done by Shahi et al and showed that alpha defensins tests showed a higher sensitivity when compared to ESR, CRP, fluid PMN and culture when detecting PJI. ${ }^{22}$ Prior use of antibiotics decreases levels of the other inflammatory markers but levels of alpha defensins remained steady without a drop in its sensitivity. The study provides use of alpha defensins as a screening test for PJI, even in the setting of prior antibiotic treatment.

Histopathology of prosthetic infections can aid in the diagnosis of CNPJIs. To do this a peri-prosthetic tissue sample must be obtained. Berbari et al reported that in 60 episodes of CNPJI, histopathologic examination of periprosthetic tissue revealed purulence in $27(45 \%)$ patients 
and acute inflammation in $47(78 \%)$ patients. ${ }^{11}$ Assessment of histology can be used in conjunction with other diagnostic measures to assess PJIs. There are currently no guidelines on the use of histopathology in assessing CNPJIs.

Synovial fluid can be tested for the presence of polymorphonuclear cell count. This has been shown to have a sensitivity of $90 \%$ and specificity of $88 \%$ to detect PJI. ${ }^{6}$ However, normal wear and tear can cause osteolysis and granulomatosis reaction. This can lead to bone resorption and loosening of prosthesis leading to a false positive, due to other causes beside infectious causes.

Imaging can aid in diagnosis of PJI by allowing visualization and assessment of the prosthesis. CT, MRIs, X-rays, fluror-2-deoxyglucose positron emission topography (FDG-PET), and scintigraphy aid in differentiating between aseptic loosening and PJIs. For example, plain radiographs can be obtained to help identify noninfectious causes such as fractures and dislocations. They can also be useful in detecting periosteal lucency, loosening of parts of prosthesis, effusion, soft tissue gas, fluid collection, and periosteal bone formation which all suggest an underlying infection but are not specific or sensitive. ${ }^{17}$ The diagnostic value of FDG-PET is controversial and not widely used in assessments of PJIs, but it has a sensitivity of $83 \%$ and specificity of $87 \% .{ }^{19}$ This modality allows visualization of inflammatory cells that utilize glucose during infections. There is also a high cost associated with its use. ${ }^{17}$ According to Tande and colleagues, three phase bone scintigraphies with a compound that is radioactively labeled, technetium-methylene diphosphonate, is one of the most widely utilized imaging techniques in the diagnosis of PJI but a limitation is its lack of specificity. ${ }^{17}$ Its specificity has been reported to be as low as $18 \%$ in PJIs that occur in one to two years after implantation and is of more use for PJI occurring late after implantation. ${ }^{17,23}$

In order to diagnose and determine the cause of PJIs, there needs to be isolation of microorganisms from reliable samples like intra-operative specimens or joint aspiration. ${ }^{5}$ Currently, culture of multiple peri-prosthetic tissue samples is the gold standard for microbiological diagnosis of $\mathrm{PJI}^{24}$ The issue with peri-prosthetic culturing is that it has a low specificity and doesn't detect an organism that grow on biofilms. A prospective study was performed by Atkins et al in which they cultured 41 prosthetic joints and found only $65 \%$ of samples from infected patients being culture positive, even in the midst of multiple peri-prosthetic samples. ${ }^{10,24}$ The use of sonication techniques can aid in providing additional diagnostic information. Sonication has been shown to increase the sensitivity of cultures by disrupting adherent bacteria, and their biofilms from surfaces of orthopedic implants. According to Legout, removed orthopedic implants are sonicated in a solution of saline to dislodge microorganisms from the surface then fluid surrounding the implant is cultured..$^{5}$ Dobbins et al performed a comparative study in which sonication cultures and swabbed cultures were obtained from an internal fixation device that was removed. The results of their study concluded that 15 of $26(58 \%)$ sonicate cultures were positive for coagulase-negative staphylococci, whereas swabbed cultures isolated coagulase-negative staphylococci in only three of 26 cultures $(12 \%) .{ }^{6,25}$ This study shows that sonication of implants yields more positive cultures when compared to traditional cultures however both techniques have low sensitivity with prior antimicrobial therapy and infection by fastidious organisms. $^{26}$

\section{DNA/PCR USAGE}

PCR is a type of nucleic acid amplification that allows the targeted portion of a genome to be detected and replicated. It provides rapid and accurate diagnosis for infections that were once viewed as idiopathic through its ability to detect DNA or RNA contained in tissues or fluids. PCR usage has not just been limited to infections but it is also a laboratory test for CNS diseases (genetic and autoimmune) and malignant neoplasms. ${ }^{26}$

Prior to usage of PCR, viral infections in the CNS were often treated with unnecessary empiric antimicrobial therapy and invasive diagnostic procedures were performed. Use of PCR has provided a way to diagnosis a multitude of infections including viral, bacterial, rickettsia, mycobacterial, and protozoal. ${ }^{26}$ Even though DNA PCR has a high sensitivity it does have its limitations. False negatives can occur under different circumstances, for example when there is delay in performing PCR or when there are inhibitors of PCR present in body fluids or tissues.

A study was performed that found usage of PCR to be highly specific and sensitive for the diagnosis of shigella and EIEC infection using stool samples. In the same study, 3 of the strains that were not originally identified using conventional techniques were identified using PCR. ${ }^{27}$ Other studies have supported these findings in various types of infections. Luna et al, observed the use of PCR to diagnose clostridium difficile infections in children hospitals. This study demonstrated that PCR gave a rapid and accurate diagnostic modality that could replace the original serial testing by enzyme-linked immunosorbent assay (EIA). ${ }^{28}$

\section{PCR USAGE IN SYNOVIAL FLUID}

Cultures of synovial fluid and intraoperative periprosthetic tissue is the standard for diagnosing PJI. The sensitivity has increased with newer techniques such as sonification of removed implants, however the infecting microorganism often remains unknown. In a study that observed the usage of both sonification of removed implants and multiplex real time PCR of sonication fluid, it was shown that the multiplex PCR can 
improve the diagnosis of PJI with specific primer. ${ }^{8,9}$ Advantages to using PCR are that even with cell non viability, fastidious growth, or previous antibiotic treatment it could still provide high sensitivity. ${ }^{29}$ Gallo et al calculated a higher sensitivity, accuracy, and negative predictive value for PCR when compared to cultured samples. Additionally, there was an $83 \%$ concordance between the result of intraoperative cultural and PCR detection of causative bacteria. ${ }^{29}$ Morgenstern et al used multiplex PCR and found that PCR was superior for detection of low virulent bacteria such as Cutibacetrium spp and coagulase negative staphylococci. They found that the short time frame, 5 hours, for PCR results compared to synovial fluid culture and growth, several days, is an important aspect when comparing PCR to culture. $^{30}$ This means that when using PCR there is no delay in identification of micro-organisms leading to appropriate treatment and prevention of adverse outcomes. In another study, a new multiplex real time PCR was used to detect methicillin resistant staphylococcus (MRS). The sensitivity and specificity of the MRS and universal PCR was $92 \%$ and $99 \%$ and $91 \%$ and $88 \%$, the concordance rate for the identification of gram +ve or gram - ve organism was $96 \%$. Based on their findings, PCR could be a use for CNPJI cases. ${ }^{31}$

Zegaer et al, compared the effectiveness of culturing versus 2 PCR methods. One PCR method used was before sequencing of the product for the identification of the species and the other was a species specific PCR for $S$. aures, $S$. epidermidis and $P$. aeruginosa. Both methods detected 16s rRNA. ${ }^{32}$ They found that the specific PCR had better results than universal, but both had issues when compared to culture. They state that PCR may not replace the gold standard of cultural because of its interpretative problems and that perhaps could be used if small amount of sample were available or when there was a negative culture after $24 \mathrm{~h}$ of incubation. ${ }^{32}$

The issue with broad range PCR is that while it has a high specificity, there is often a poor sensitivity in diagnosing infections. $^{33}$ However when the PCR and bacterial cultural were considered together, the sensitivity of PCR increased to $67 \%$. Additionally in another study that used a method of using MRSA for fast detection of methicillin resistant coagulase negative Staphylococci in PJI, it was found that it lead to early microbial diagnosis but with low sensitivity and high cost. ${ }^{34}$ In a meta-analysis that looked at several studies for the validity of PCR it was found that the sensitivity of $86 \%$ and specificity of $91 \%$ make it practical for clinical use. ${ }^{35}$

\section{CONCLUSION}

While PCR could provide a better diagnostic approach to PJI, more studies need to be done at the time to ascertain if this tool would be useful in the acute management of PJI. Some of the issues that still need to be addressed include the number of pathogens isolated or detected by this test leaving one unsure as to which is the primary pathogen that caused the infection. Over use of antibiotics in these situations could eventually lead to a higher risk of clostridium difficle infections as well. Therefore, the need to determine faster methods of identifying the causes of infections is crucial to avoid the inappropriate use of antibiotics and reduce complications associated with PJIs.

\section{Funding: No funding sources \\ Conflict of interest: None declared \\ Ethical approval: Not required}

\section{REFERENCES}

1. Losina E, Katz JN. Total joint replacement outcomes in patients with concomitant comorbidities: a glass half empty or half full? Arthritis Rheumatism. 2013;65(5):1157-9.

2. Beswick AD, Wylde V, Gooberman-Hill R, Blom A, Dieppe P. What proportion of patients report long-term pain after total hip or knee replacement for osteoarthritis? A systematic review of prospective studies in unselected patients. BMJ Open. 2012;2(1):e000435.

3. Skinner H, McMahon P. Current Diagnosis \& Treatment in Orthopedics. 5th ed. McGraw Hill Publication. 2014.

4. Crawford RW, Murray DW. Total hip replacement: Indications for surgery and risk factors for failure. Annals of Rheumatic Diseases. 1997;56(8):445-57.

5. Legout L, Senneville E. Periprosthetic Joint Infections: Clinical and Bench Research. Sci World J. 2013;1-17.

6. Yoon PW, Lee YK, Ahn J, Jang EJ, Kim Y, Kwak $\mathrm{HS}$, et al. Epidemiology of hip replacements in Korea from 2007 to 2011. J Korean Med Sci. 2014;29:852-8.

7. Drago L, De Vecchi E, Bortolin M, Zagra L, Romano C, Cappelletti, L. Epidemiology and Antibiotic Resistance of Late Prosthetic Knee and Hip Infections. J Arthroplast. 2017;32(8) 2496-500.

8. Parikh, MS, Antony, S. A comprehensive review of the diagnosis and management of prosthetic joint infections in the absence of positive cultures. J Infection Public Health. 2016;9:545-56.

9. Nair PK, Bhat VG, Vaz MS. Prosthetic joint infections-a clinico-microbiological perspective: Review article. World J Clin Infectious Diseases. 2014;4(3):9-15.

10. 10 Atkins BL, Athanasou N, Deeks JJ, Crook DW, Simpson H, Peto TE, et al. Prospective evaluation of criteria for microbiological diagnosis of prostheticjoint infection at revision arthroplasty. The OSIRIS Collaborative Study Group. J Clin Micro. 1998;36:2932-9.

11. Beribari EF, Marculescu C, Sia I, Hanssen AD, Steckelberg JM, Gullerud R, Osmon DR. CultureNegative Prosthetic Joint Infection, Clinical Infectious Diseases. 2007;45(9):1113-9.

12. Wu C, Qu X, Liu F, Li H, Mao Y, Zhu Z. Risk Factors for Periprosthetic Joint Infection after Total Hip Arthroplasty and Total Knee Arthroplasty in Chinese Patients. PLoS ONE. 2014;9(4):e95300. 
13. Peel TN, Cheng AC, Buising KL, Choong PF. Microbiological etiology, epidemiology, and clinical profile of prosthetic joint infections: are current antibiotic prophylaxis guidelines effective? Antimicrobial Agents and Chemotherapy. 2012;56:2386-239.

14. Peel TN, Dowsey MM, Daffy JR, Stanley PA, Choong PF, Buising KL. Risk factors for prosthetic hip and knee infections according to arthroplasty site. J Hospital Infection. 2011;79(2):129-33.

15. Mraovic B, Suh D, Jacovides C, Parvizi J. Perioperative hyperglycemia and postoperative infection after lower limb arthroplasty. J Diabetes Sci Tech. 2011;5(2):412-8.

16. Seneviratne CJ, Yip JW, Chang JW, Zhang CF, Samaranayake LP. Effect of culture media and nutrients on biofilm growth kinetics of laboratory and clinical strains of Enterococcus faecalis. Archives of Oral Biology. 2013;58:1327-34.

17. Tande AJ, Patel R. Prosthetic Joint Infection. Clinical Microbiology Reviews. 2014;27(2):302-45.

18. Malekzadeh D, Osmon DR, Lahr BD, Hanssen AD, Berbari EF. Prior use of antimicrobial therapy is a risk factor for culture negative prosthetic joint infection. Clinical Orthopaedics and Related Research. 2010;468(8):2039-45.

19. Osmon DR ,Berbari EF, Berendt AR, Lew D, Zimmerli W, Steckelberg JM, et al. Executive summary: diagnosis and management of prosthetic joint infection: clinical practice guidelines by the Infectious Diseases Society of America. Clinical Infectious Disease. 2013;56:1-10.

20. Barrett L, Atkins B. The clinical presentation of prosthetic joint infection. $\mathrm{J}$ Antimicrobial Chemotherapy. 2014;69(1):i25-7.

21. Ganz T, Selsted ME, Szklarek D, Harwig SS, Daher K, Bainton DF, et al. Natural peptide antibiotics of human neutrophils. J Clin Invest. 1985;76:1427-35.

22. Shahi A, Kheir MM, Tarabichi M, Hosseinzadeh HR, Tan TL, Parvizi J. Serum D-Dimer Test Is Promising for the Diagnosis of Periprosthetic Joint Infection and Timing of Reimplantation. J Bone Joint Surg. 2017;99(17):1419-27.

23. Magnuson JE, Brown ML, Hauser MF, Berquist TH, Fitzgerald RH, Klee GG. In-111-labeled leukocyte scintigraphy in suspected orthopedic prosthesis infection: comparison with other imaging modalities. Radiology. 1988;168:235-9.

24. Street L, Sanderson ND, Atkins BL, Brent AJ, Cole $\mathrm{K}$, Foster D, et al. Molecular Diagnosis of Orthopedic-Device-Related Infection Directly from Sonication Fluid by Metagenomic Sequencing. J Clin Micro. 2017;55(8);2334-47.

25. Dobbins JJ, Seligson D, Raff MJ. Bacterial colonization of orthopedic fixation devices in the absence of clinical infection. $J$ Infectious Dis. 1988;158:203-5.

26. DeBiasi RL, Tyler KL. Polymerase Chain Reaction in the Diagnosis and Management of Central
Nervous System Infections. Achieve of Neurology. 1999;56(10):1215-9.

27. Dutta S, Chatterjee A, Rajendran K, Roy S, Pramanik KC, Bhattachary SK. Sensitivity and performance characteristics of a direct PCR with stool samples in comparison to conventional techniques for diagnosis of Shigella and enteroinvasive Escherichia coli infection in children with acute diarrhea in Calcutta, India. J Med Micro. 2001;50(8):667-4.

28. Luna R, Boyanton B, Mehta S, Courtney E, Webb C, Revell, et al. Rapid Stool- Based Diagnosis of Clostridium difficile Infection by Real-Time PCR in Children's Hospital. J clin Microbiol. 2011;49(3):851-7.

29. Gallo J, Dendis M, Loveckova Y, Sauer P, Zapletalova J, Koukalova D. Culture and PCR analysis of joint fluid in the diagnosis of prosthetic joint infection. New Microbiologica. 2008;31:97104.

30. Morgenstern C, Cabric S, Perka C, Trampuz A, Renz N. Synovial fluid multiplex PCR is superior to culture for detection of low-virulent pathogens causing periprosthetic joint infection. Diagnostic Microbiology and Infectious Disease. 2018;90(2):115-9.

31. Kawamura M, Kobayashi N, Inaba Y, Choe H, Tezuka T, Kubota S, et al. A new multiplex realtime polymerase chain reaction assay for the diagnosis of periprosthetic joint infection. Modern Rheumatology. 2017;27(6):1072-8.

32. Zegaer BH, Ioannidis A, Babis GC, Ioannidou V, Kossyvakis A, Bersimis S, et al. Detection of Bacteria Bearing Resistant Biofilm Forms, by Using the Universal and Specific PCR is Still Unhelpful in the Diagnosis of Periprosthetic Joint Infections. Frontiers of Medicine. 2014;1:30.

33. Man FH, Graber P, Lüem M, Zimmerli W, Ochsner PE, Sendi P. Broad-Range PCR in Selected Episodes of Prosthetic Joint Infection. Infection. 2009;37(3):292-4.

34. Lourtet-Hascoëtt J, Bicart-See A, Félicé M, Giordano G, Bonnet E. Is Xpert MRSA/SA SSTI real-time PCR a reliable tool for fast detection of methicillin-resistant coagulase-negative staphylococci in periprosthetic joint infections? Diagnostic Microbiology and Infectious Disease. 2015;83(1):59-62.

35. Qu X, Zhai Z, Li H, Liu X, Zhu Z, Dai K. PCRBased Diagnosis of Prosthetic Joint Infection. J Clin Microbiol. 2013;51(8):2742-6.

Cite this article as: Ogunleye $\mathrm{T}$, de Leon MP, Antony SJ. The use of polymerase chain reaction in the diagnosis and management of prosthetic joint infections: a debatable issues at this time. Int J Res Orthop 2019;5:362-7. 